\title{
Chimerization of multiple antibody classes using splice overlap extension PCR
}

\author{
Martina L. Jones and Ross T. Barnard \\ The University of Queensland, St. Lucia, QLD, Australia
}

BioTechniques 38:181-182 (February 2005)

Splice overlap extension (SOE) PCR allows the fusion of two sequences of DNA without the use of restriction enzymes (1). Briefly, SOE joins two sequences that have regions of short sequence complementarity between the $3^{\prime}$ end of the first sequence and the $5^{\prime}$ end of the other. The sequences are fused during PCR, as the overlapping sequences will hybridize and extend to produce a fulllength chimeric sequence. Sequence complementarity at the fusion site can be introduced into two unrelated fragments by incorporating common sequence tags into primers in a firstround PCR. Conversely, if the two fragments already contain sequence similarity at the fusion point, then a chimeric product can be produced without modification of the sequence.

The J-regions of immunoglobulin genes of mouse and human have high similarity, allowing the fusion of a mouse variable region with a human constant region without the addition of complementary tags. A chimeric antibody has previously been produced by SOE utilizing J-region similarity (2), but the primer sequence only allowed amplification of and chimerization with the constant region of immunoglobulin $\mathrm{G}$ (IgG). Here we report primer sequences, directed to the J-regions of light and heavy chain immunoglobulins, which allow both the isolation of variable regions from mouse hybridoma cDNA and the isolation of constant regions of isotypes $\operatorname{IgG}, \operatorname{IgM}$, and $\operatorname{IgA}$ from human blood. The J-region primers for the variable and the constant regions are reverse complementary, allowing subsequent fusion of the sequences by SOE (see Figure 1). This allows for a very simple isolation and chimerization procedure with versatility to allow the fusion of the same variable region to any constant region.

The J-region primers were designed from a sequence alignment of known mouse and human alleles contained in the International ImMunoGeneTics database (3). Human heavy chain constant regions for $\operatorname{IgG}, \operatorname{IgM}$, and $\operatorname{IgA}$ were each amplified from peripheral blood lymphocyte cDNA using the same forward primer $\left(\mathrm{J}_{\mathrm{H}} \mathrm{F}\right)$ and the class-specific reverse primers, IgG_R, IgM_R, and IgA_R, respectively. The $\bar{\kappa}$ chain constant region was amplified using $\mathrm{J} \kappa \mathrm{F}$ and Igא_R. Primer sequences for the amplification of constant regions are given

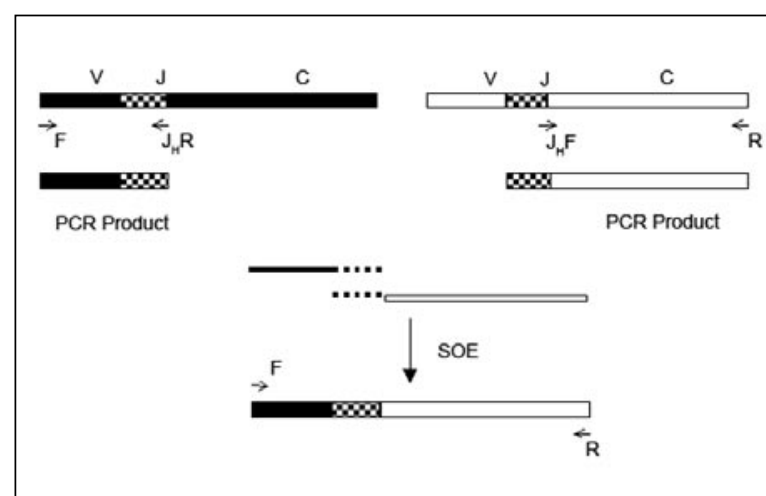

Figure 1. Chimerization of mouse (black) and human (white) immunoglobulin sequences by splice overlap extension (SOE) PCR. The mouse variable $(V)$ region is amplified with primers $F$ and $J_{H} R$, and the human constant $(C)$ region is amplified with primers $\mathrm{J}_{\mathrm{H}} \mathrm{F}$ and R. Primers $J_{H} R$ and $J_{H} F$ are reverse complementary and are specific to a part of the J-region (hashed), which has sequence homology between mouse and human. When mixed together, PCR products will hybridize and extend from the splice overlap site, and the chimeric product is amplified from primers $\mathrm{F}$ and $\mathrm{R}$. in Table 1. cDNA was produced from a hybridoma secreting antibodies against Orientia tsutsugamushi (Rickettsia scrub typhus), and variable regions were amplified using a set of degenerate forward primers (4) and reverse primers $J_{H} R$ and $J \kappa R$ for the heavy chain and $\kappa$ chain, respectively. The PCR consisted of 1 U Platinum ${ }^{\circledR}$ Pfx DNA polymerase (Invitrogen, Carlsbad, CA, USA), $1 \times P f x$ Amplification buffer (Invitrogen), $1.5 \mathrm{mM}$ $\mathrm{MgSO}_{4}, 0.2 \mathrm{mM}$ dNTP, $1 \mu \mathrm{M}$ each of forward and reverse primer, and approximately $50 \mathrm{ng}$ cDNA. PCR cycling conditions were as follows: initial denaturation at $94^{\circ} \mathrm{C}$ for $2 \mathrm{~min}$, followed by 25 cycles of denaturation at $94^{\circ} \mathrm{C}$ for $1 \mathrm{~min}$, annealing at $50^{\circ} \mathrm{C}$ for constant regions or $60^{\circ} \mathrm{C}$ for variable regions for $30 \mathrm{~s}$, and extension at $72^{\circ} \mathrm{C}$ for $2 \mathrm{~min}$. The reaction was completed with a final extension at $72^{\circ} \mathrm{C}$ for $10 \mathrm{~min}$. Products were cloned into $\mathrm{pCR}^{\circledR} 2.1$-TOPO ${ }^{\circledR}$ (Invitrogen).

PCR products derived from variable and constant region coding sequences were reamplified from the storage plasmid, electrophoresed, and then extracted and cleaned to remove excess J-region primers. Approximately 0.2 pmol each of variable region and constant region products were mixed, and the same PCR was repeated using the variable region forward primer and the constant region reverse primer. In this reaction, the two templates

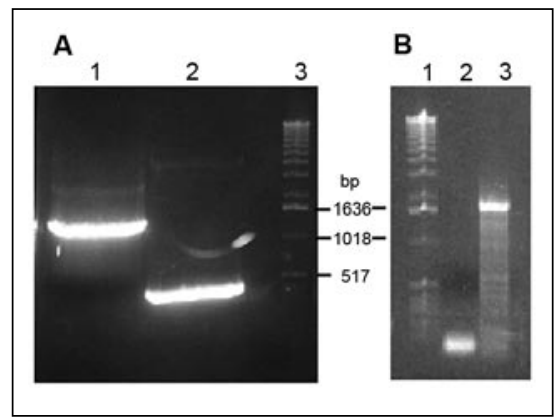

Figure 2. Construction of chimeric mouse-human M-class heavy chain by splice overlap extension (SOE). (A) PCR amplification of templates. Lane 1, human immunoglobulin M (IgM) constant region (1394 bp); lane 2, mouse heavy chain variable region (411 bp); lane 3, Molecular Weight Marker X (Roche Applied Science, Sydney, Australia). (B) SOE. Lane 1, Molecular Weight Marker X; lane 2, negative control (no template); lane 3, mouse-human chimeric IgM heavy chain (1772 bp). 
Table 1. Primer Sequences

\begin{tabular}{|ll|}
\hline Primer & Sequence \\
\hline $\mathrm{J}_{\mathrm{H}} \mathrm{F}$ & 5'-GGGGCCAAGGGACCATGGTCACCGTCTCCTCAG-3' $^{\prime}$ \\
$\mathrm{J} \kappa \mathrm{F}$ & 5'-GGGACCAAGCTGGAAATCAAACG-3' $^{\prime}$ \\
IgG_R & 5'-TCATTTACCCGGAGACAGGGAG-3' $^{\prime}$ \\
IgM_R & 5'-TCAGTAGCAGGTGCCAGCTGTGTCGG-3' $^{\prime}$ \\
IgA_R & 5'-TCAGTAGCAGGTGCCGTCCACCTCCGCC-3' $^{\prime}$ \\
Igא_R & 5'-CTCCCTCTAACACTCTCCCCTG-3' $^{\prime}$ \\
\hline
\end{tabular}

Table 2. ELISA Readings of Supernatants from Cultures Transfected with Constructs Containing Chimeric IgG, IgA, or IgM Antibodies

\begin{tabular}{|lccc|}
\hline & $\begin{array}{c}\text { Anti-IgG } \\
\text { Conjugate }\end{array}$ & $\begin{array}{c}\text { Anti-IgA } \\
\text { Conjugate }\end{array}$ & $\begin{array}{c}\text { Anti-IgM } \\
\text { Conjugate }\end{array}$ \\
\hline Negative Control & 0.091 & 0.105 & 0.085 \\
Positive Control & 1.147 & N.A. & 0.917 \\
IgG Transfection & 0.869 & 0.074 & 0.074 \\
IgA Transfection & 0.072 & 0.302 & 0.076 \\
IgM Transfection & 0.076 & 0.080 & 0.185 \\
Results show absorbance at 450 nm after using horseradish peroxi- \\
dase (HRP)-conjugated antibodies and tetramethylbenzidine substrate. \\
A positive control for IgA was unavailable. ELISA, enzyme-linked immu- \\
nosorbent assay; Ig, immunoglobulin; N.A., not applicable. \\
\hline
\end{tabular}

hybridize, and a chimeric product is obtained by SOE (see Figure 2). The chimeric heavy chain and $\kappa$ chain were each cloned into pCR 2.1-TOPO, then excised using vector-derived restriction enzyme sites, and then both ligated into the bicistronic expression vector pBudCE4.1 (Invitrogen). This vector was transfected using Lipofectamine ${ }^{\mathrm{TM}} 2000$ (Invitrogen) into $\mathrm{CHO}$ cells. Supernatants from 48-h cultures were tested for antibody functionality in a Rickettsia scrub typhus enzyme-linked immunosorbent assay (ELISA) kit (Panbio, Brisbane, QLD, Australia). Functional antibody binds to immobilized antigen and is detected with horseradish peroxidase (HRP)-conjugated anti-human IgG, IgM, or IgA. Positive controls are human serum containing antibodies against the scrub typhus antigen, but no IgA control was available, as an IgA kit for scrub typhus is not produced by the manufacturer. As shown in Table 2, functional antibodies were produced, with no cross-reactivity with HRP conjugates. Also, the supernatant from an IgG culture was nonreactive in a Dengue-specific ELISA kit, indicating specificity of the antibody towards the scrub typhus antigen (data not shown).
The J-region primers and the SOE procedure have been successfully used for isolation of variable regions from hybridomas specific for Bordetella pertussis (whooping cough) and Rickettsia rickettsii (Rocky Mountain spotted fever) and chimerization of the anti- $B$. pertussis antibodies (data not shown).

In summary, the sequence complementarity at the $3^{\prime}$ end of mouse and human immunoglobulin J-regions allows the simple production of chimeric mouse-human monoclonal antibodies by SOE PCR, avoiding the use of restriction enzymes. The J-region primers were designed to allow isolation of variable regions from mouse hybridomas and constant regions from human blood. The variable region can then be fused to any constant region of classes $\mathrm{IgG}$, $\operatorname{IgM}$, and $\operatorname{IgA}$.

\section{ACKNOWLEDGMENTS}

This work was supported by a linkage grant from the Australian Research Council and PANBIO Ltd, Windsor, QLD, Australia. We are grateful to $M s$. Carmel George and Ms. Tomoko Nagasaki for the production and supply of hybridomas.

\section{COMPETING INTERESTS STATEMENT}

The authors declare no competing interests.

\section{REFERENCES}

1.Horton, R.M., H.D. Hunt, S.N. Ho, J.K. Pullen, and L.R. Pease. 1989. Engineering hybrid genes without the use of restriction enzymes: gene splicing by overlap extension. Gene 77:61-68

2.Stewart, L.M., S. Young, G. Watson, S.J. Mather, P.A. Bates, H.A. Band, R.W. Wilkinson, E.L. Ross, and D. Snary. 1999. Humanisation and characterisation of PR1A3, a monoclonal antibody specific for cell-bound carcinoembryonic antigen. Cancer Immunol. Immunother. 47:299-306.

3.Lefranc, M.P. 2003. IMGT, the international ImMunoGeneTics database. Nucleic Acids Res. 31:307-310.

4.Coloma, M.J., A. Hastings, L.A. Wims, and S.L. Morrison. 1992. Novel vectors for the expression of antibody molecules using variable regions generated by polymerase chain reaction. J. Immunol. Methods 152:89-104.

Received 19 July 2004; accepted 23 September 2004.

Address correspondence to Ross T. Barnard, School of Molecular and Microbial Sciences, University of Queensland, St. Lucia, QLD 4072, Australia. e-mail: rossbarnard@uq.edu.au 